\title{
Tivozanib for the treatment of renal cell carcinoma: patient selection and perspectives
}

This article was published in the following Dove Press journal:

International Journal of Nephrology and Renovascular Disease

\section{RJ Rodenburg \\ FALM Eskens}

Erasmus MC Cancer Institute, Department of Medical Oncology, Rotterdam, The Netherlands
Correspondence: RJ Rodenburg Erasmus MC Cancer Institute, Location NT5, PO Box 2040, Rotterdam 3000 CA, The Netherlands

Tel +3 II07040019

Fax +3 II07041003

Email r.rodenburg@erasmusmc.nl

\begin{abstract}
Tivozanib is an oral selective vascular endothelial growth factors receptor (VEGFR) tyrosine kinase inhibitor that is recently approved by the European Medicines Agency for the treatment of previously untreated patients with metastatic renal cell carcinoma (mRCC) as well as for those patients with disease progression during or after cytokine therapy. Nowadays, in first-line and second-line treatment of $\mathrm{mRCC}$, there is an abundance of options, mainly consisting of VEGFR-directed tyrosinekinase inhibitors. This review focusses on the role of tivozanib with respect to patient selection and future perspectives in this fast-changing landscape.
\end{abstract}

Keywords: tivozanib, metastatic renal cell cancer, tyrosine kinase inhibitor

\section{Introduction}

Renal cell cancer (RCC) is the $7^{\text {th }}$ most common cancer in the UK with an increasing incidence of up to 12,600 new cases per year in $2015 .{ }^{1}$ Being related to lifestyle factors such as obesity, smoking, hypertension, and increasing age, RCC has been one of the fastest increasing cancers in the past decades. ${ }^{2}$ Males are more frequently affected than females and the peak incidence is at 60-80 years. The most frequently diagnosed histological subtype is clear cell RCC (80\%) followed by papillary (10-15\%) and chromofobe (5-10\%) RCC. Due to its location, RCC initially goes often unnoticed, and as a result, most patients present with either locally advanced or metastatic disease. About one-third of patients presenting with RCC have metastatic disease (metastatic renal cell carcinoma (mRCC)) at their time of diagnosis. ${ }^{3}$ In contrast to the situation of locally advanced disease, where a radical nephrectomy is a potentially curative option, performing a nephrectomy in case of metastatic disease does not seem to be the golden standard anymore. ${ }^{4}$ Before considering a systemic treatment for $\mathrm{mRCC}$, it is crucial to consider that in many patients $\mathrm{mRCC}$ can have a very indolent course, meriting close observation as a viable and rational first-line treatment option. As a general finding, $\mathrm{mRCC}$ is insensitive to either hormonal and cytotoxic therapies, but blocking the intracellular signalling activity of vascular endothelial growth factors receptors (VEGFR) through tyrosinekinase inhibitors (TKI) and thereby inhibiting angiogenesis has been shown to be an effective standard of care. ${ }^{5}$ Inhibiting the mammalian target of rapamycin (mTOR), a kinase protein which is important in signal transduction of factors associated with angiogenesis and proliferation, has for years been considered another rational target for treatment, but nowadays this paradigm is rapidly losing terrain. The standard of care in advanced or mRCC in essence depends on the risk stratification according to the Memorial Sloan Kettering Cancer Center and/or 
International Metastatic RCC Database consortium criteria. ${ }^{6,7}$ Until recently, first-line therapy in patients with good or intermediate prognosis mRCC usually consisted of a VEGFR targeting TKI such as pazopanib or sunitinib or alternatively the combination of bevacizumab with IF8N$\alpha^{8-11}$ For patients with poor prognosis mRCC, first-line treatment with the mTOR inhibitor temsirolimus was recommended, even though sunitinib, sorafenib, and pazopanib were frequently used alternatives. ${ }^{12}$ Recently, a large randomized phase III study, however, has unequivocally shown that the combination of nivolumab and ipilimumab was superior to sunitinib with regard to the primary end point overall survival in patients with intermediate- and poor-risk mRCC, but not in good-risk patients. ${ }^{13}$ Based on this study, the updated ESMO 2019 guidelines prefer this combination as first-line treatment in patients with intermediate- and poorrisk mRCC. ${ }^{14}$ Whether the recent publications of the combination of either pembrolizumab or avelumab with the TKI axitinib will again change the current (and seemingly evermoving) landscape of first-line treatment of mRCC remains to be established. ${ }^{15,16}$ Second-line treatment in patients with progressive disease either during or after first-line treatment depends on a variety of factors. For patients with disease progression during or after first-line cytokine treatment, second-line therapy usually consists of singleagent TKI treatment, where sorafenib, tivozanib, or axitinib can be considered. ${ }^{11,14,17-19}$ In case of disease progression during or after first-line TKI treatment, a variety of treatment options is available, whereby either nivolumab or cabozantinib have compelling data regarding effects on overall survival. $^{20,21}$ If these options cannot be considered, lenvatinib combined with everolimus could be an option, albeit that their effect on the primary end point progression-free survival is based upon randomized phase II clinical data. ${ }^{18,22}$ There is no standard recommendation for third-line treatment; hence, these patients should preferably be enrolled into clinical trials to create more evidence for TKI or immunotherapy in third or fourth line. ${ }^{23-25}$ Even though the prognosis for patients with advanced or $\mathrm{mRCC}$ has significantly improved over the last one to two decades following the introduction of the above-mentioned treatment options, there still remains a need for more effective and (better) tolerable treatment options in the various lines of treatment.

\section{Tivozanib}

In August 2017, the European Medicines Agency (EMA) approved tivozanib, a highly selective VEGFR TKI for treatment of $\mathrm{mRCC}$ patients who were previously untreated or in whom the disease progression occurred after one prior treatment with cytokine therapy. ${ }^{26}$

\section{Mode of action}

Functional preclinical trials with tivozanib showed a selective and potent inhibition of VEGF tyrosine kinase receptors 1,2 , and $3 .^{27}$ The VEGF signaling pathway plays an important role in physiological and pathological conditions such as endothelial cell proliferation, migration, and survival and thus angiogenesis, which facilitates tumor growth and the formation of metastases. There are three VEGF receptors $(1,2$, and 3 ) and five VEGF ligands (A, B, C, D, and placental growth factor). Each ligand exhibits distinct but overlapping binding profiles for the three receptors. VEGFR-1 is critical for vessel morphogenesis and modulation of endothelial cell proliferation, whereas VEGFR-3 promotes vascular network formation and endothelial sprouting. The predominant receptor for endothelial cell proliferation and migration however is VEGFR-2. Most tumors produce VEGF, and in preclinical models, tumor growth was significantly reduced through inhibition of VEGF-induced angiogenesis. ${ }^{28}$ As each VEGFR plays an important and different role in cancer angiogenesis, it may be critical to block all three VEGFRs. Tivozanib is a TKI with the capacity to inhibit all three VEGFRs.

\section{Trials with Tivozanib}

Eskens et al and Niwakawa et al performed phase I clinical studies in patients with advanced solid tumors and showed that $1.5 \mathrm{mg}$ of tivozanib once daily in an on/off treatment schedule was well tolerated. ${ }^{29,30}$ The best overall response observed in these studies was stable disease, sometimes lasting over 6 months. The most frequently observed severe adverse events (AEs) (grade $\geq 3$ ) included hypertension, proteinuria, hand-foot skin reaction, fatigue, diarrhea, and hepatic function abnormalities.

Subsequently, clinical activity of tivozanib in patients with $\mathrm{mRCC}$ was demonstrated in a phase II study of Nosov et al. ${ }^{31}$ Patients with $\mathrm{mRCC}$ without prior targeted therapy received tivozanib $1.5 \mathrm{mg}$ orally for 3 weeks followed by 1 week off-drug. The overall response rate (ORR) was $24 \%$ and the median progression-free survival (PFS) was 11.7 months. Most patients (83\%) had a clear cell histology, and in patients with clear cell histology who had previously undergone a nephrectomy, the ORR was $30 \%$ and a median (PFS) of 14.8 months was observed. 
Subsequently, Motzer et al conducted a large international open-label, randomized, multicenter phase III trial comparing the efficacy and tolerability of tivozanib versus that of sorafenib in patients with clear cell mRCC. Additional inclusion criteria were an ECOG performance status (PS) of $0-1$, a prior nephrectomy, and $0-1$ prior lines of treatment, which were not to have consisted of inhibitors of mTOR or VEGF. ${ }^{19}$ The primary end point of this study, which enrolled 517 patients, was PFS. The study groups were well-balanced, except for PS (tivozanib group $45 \%$ of the patients had a PS of 0 , while in the sorafenib group this was $54 \%, p=0,035)$. Patients were stratified for either 0 or 1 previous line of treatment; in both groups, $70 \%$ of the patients were treatment naive. In the intention to treat population, the primary end point median PFS was 11.9 months (95\%-CI: 9.3-14.7) in the tivozanib group and 9.1 months $(95 \%$-CI: $7.3-9.5)$ in the sorafenib group (HR: 0.797 [95\%-CI: 0.639-0.993]; $p=0.042)$ ). In the prospectively defined subgroup of patients without previous treatment, PFS was 12.7 months (95\%-CI: 9.1-15) in the tivozanib group and 9.1 months (95\%-CI: 7.3-10.8) in the sorafenib group (HR: 0.756 [95\%-CI: $0.580-0.985] ; \quad p=0.037)$. The median OS, a secondary end point in this study, was not significantly different (29.3 vs 28.8 months, HR 1.245, $p=0.105$ ) because crossover from sorafenib to tivozanib was allowed and patients received more third-line therapy after sorafenib than after tivozanib due to geographical reasons. AEs more frequently observed with tivozanib than with sorafenib were dysphonia and hypertension, whereas patients treated with sorafenib experienced more hand-foot skin reaction and diarrhea. Overall, patients receiving tivozanib needed less dose reduction due to AEs than patients receiving sorafenib.

\section{Tivozanib as treatment option}

As mentioned, the landscape for patients with mRCC has changed tremendously over the last 10-15 years, with VEGFR-directed TKIs, inhibitors of mTOR, and more recently immunotherapy as emerging treatment options. However, despite this continuously expanding plethora of available treatment options, $\mathrm{mRCC}$ is still not a curable disease. Treatment focuses on extending survival while maintaining the quality of life. Unfortunately, all treatment options have side effects which often force adjustments or discontinuation of treatment. Therefore, and again taking into consideration that in some patients mRCC can run an indolent course over prolonged periods of time, meriting a period of observation before systemic treatment indeed should be considered, a careful judgment of efficacy and toxicity should be made before any treatment is initiated.

Nowadays, in first- and second-line treatment of mRCC, there is an abundance of options. The available evidence for immunotherapy in first and second line and cabozantinib in second line caused a paradigm shift. However, the VEGFR-directed TKIs such as sunitinib, sorafenib, pazopanib, lenvatinib, axitinib, and tivozanib are still viable and proven treatment options. Most of these TKIs have been compared in randomized phase III studies with either interferon alpha or placebo, with only a limited number of randomized studies comparing two TKIs. ${ }^{8,18,19,32}$ Therefore, evaluating their relative efficacy is difficult. Side effects are mainly a class effect; hence, all TKIs induce AEs but with different degrees of severity. The side-effect profile of the VEGFR selective TKI tivozanib seems to differ somewhat from that of most of the other TKIs and mainly consists of dysphonia and hypertension, while the more cumbersome side effects such as skin toxicities and hand-foot skin reactions were only infrequently observed. ${ }^{19,30,31}$

It has been somewhat puzzling that although the randomized phase III trial with tivozanib as first- or second-line treatment for patients with $\mathrm{mRCC}$ was already published in 2013, EMA only recently approved tivozanib for this indication. Probably, the fact that FDA rejected tivozanib for this indication following the request for registration in the USA in 2013 explains this delay. In the USA, a large randomized phase III study comparing tivozanib to sorafenib in patients with refractory $\mathrm{mRCC}$ has been closed for accrual and first results are expected to become available in the next couple of months. It can be anticipated that if results from this study are favorable, resubmission of tivozanib to FDA could be pursued.

\section{Perspectives}

Despite the large and expanding arsenal of treatment options, mRCC remains an incurable disease. This prompts investigators to combine various classes of agents in an attempt to improve treatment outcomes while maintaining at least an acceptable quality of life. Within this framework, tivozanib combination therapy with 
temsirolimus has been explored, revealing that this combination could be safely given to patients with $\mathrm{mRCC}$ previously exposed to at least one line of VEGFR-directed therapy. ${ }^{33}$ Even though confirmed partial responses and a high percentage of disease stabilization was observed, no follow-up studies have been initiated. A single-arm phase Ib/II study combining tivozanib with nivolumab (TiNivo trial) has been closed for accrual and results are also expected within the next couple of months (NCT03136627).

\section{Patient selection}

Pending the FDA approval of tivozanib, treatment options for metastatic renal cancer are emerging rapidly. With its specific side-effect profile of mainly hypertension and hoarseness, tivozanib is a relatively mild and welltolerated TKI. Therefore, tivozanib might be an interesting treatment option for patients who suffer from severe side effects of other TKIs or have a decreased Karnofsky Performance Score. Although immunotherapy is an emerging treatment option for mRCC, not all patients are eligible for this treatment. For instance, patients with severe autoimmune disease are unsuitable for immunotherapy. TKIs such as tivozanib therefore remain an important treatment option. Furthermore, we eagerly await clinical data on the effect of tivozanib in the third line.

\section{Conclusions and future perspectives}

Tivozanib is an oral selective VEGFR-TKI that has demonstrated antitumor activity and effects on overall survival in various studies in patients with mRCC. The most common AEs are manageable hypertension and hoarseness. Recently, the EMA has approved tivozanib for the treatment of previously untreated patients with $\mathrm{mRCC}$ as well as for those patients who had disease progression during or after cytokine therapy. Therefore, tivozanib has now become another treatment option for these patients. Because of the crowded and rapidly changing field of treatment options for patients with $\mathrm{mRCC}$, further research is ongoing to determine the role of tivozanib within this field. Special emphasis is currently put on the combination of TKIs such as tivozanib and other yet approved options for patients with mRCC, in particular, anti-PD-(L)1-directed monoclonal antibodies.

\section{Disclosure}

The authors report no conflicts of interest in this work.

\section{References}

1. Kidney Cancer Statistics. Cancer Research UK.

2. Chow WH, Dong LM, Devesa SS. Epidemiology and risk factors for kidney cancer. Nat Rev Urol. 2010;7:245-257. doi:10.1038/ nrurol.2010.46

3. SEER Stat Fact Sheets: Kidney and Renal Pelvis

4. Mejean A, Ravaud A, Thezenas S, et al. Sunitinib alone or after nephrectomy in metastatic renal-cell carcinoma. $N$ Engl $\mathrm{J}$ Med. 2018;379:417-427. doi:10.1056/NEJMoa1803675

5. Motzer RJ, Russo P. Systemic therapy for renal cell carcinoma. J Urol. 2000;163:408-417.

6. Heng DY, Xie W, Regan MM, et al. Prognostic factors for overall survival in patients with metastatic renal cell carcinoma treated with vascular endothelial growth factor-targeted agents: results from a large, multicenter study. J Clin Oncol. 2009;27:5794-5799. doi: $10.1200 /$ JCO.2008.21.4809

7. Ko JJ, Xie W, Kroeger N, et al. The international metastatic renal cell carcinoma database consortium model as a prognostic tool in patients with metastatic renal cell carcinoma previously treated with first line targeted therapy: a population-based study. Lancet Oncol. 2015;16:293-300. doi:10.1016/S1470-2045(14)71222-7

8. Motzer RJ, Hutson TE, Cella D, et al. Pazopanib versus sunitinib in metastatic renal-cell carcinoma. N Engl J Med. 2013;369:722-731. doi:10.1056/NEJMoa1303989

9. Motzer RJ, Hutson TE, Tomczak P, et al. Sunitinib versus interferon alfa in metastatic renal-cell carcinoma. $N$ Engl $J$ Med. 2007;356:115-124. doi:10.1056/NEJMoa065044

10. Escudier B, Pluzanska A, Koralewski P, et al. Bevacizumab plus interferon alfa-2a for treatment of metastatic renal cell carcinoma: a randomised, double-blind phase III trial. Lancet. 2007;370:2103-2111. doi:10.1016/S0140-6736(07)61904-7

11. Sternberg CN, Davis ID, Mardiak J, et al. Pazopanib in locally advanced or metastatic renal cell carcinoma: results of a randomized phase III trial. J Clin Oncol. 2010;28:1061-1068. doi:10.1200/JCO.2009.23.9764

12. Hudes G, Carducci M, Tomczak P, et al. Temsirolimus, interferon alfa, or both for advanced renal-cell carcinoma. $N$ Engl J Med. 2007;356:2271-2281. doi:10.1056/NEJMoa066838

13. Motzer RJ, Tannir NM, McDermott DF, et al. Nivolumab plus ipilimumab versus sunitinib in advanced renal-cell carcinoma. $N$ Engl J Med. 2018;378:1277-1290. doi:10.1056/NEJMoa1712126

14. Escudier B, Porta C, Schmidinger M, et al. Renal cell carcinoma: ESMO clinical practice guidelines for diagnosis, treatment and follow-up. Ann Oncol. 2019. doi:10.1093/annonc/mdz056

15. Rini BI, Plimack ER, Stus V, et al. Pembrolizumab plus axitinib versus sunitinib for advanced renal-cell carcinoma. $N$ Engl J Med. 2019;380:1116-1127. doi:10.1056/NEJMoa1816714

16. Motzer RJ, Penkov K, Haanen J, et al. Avelumab plus axitinib versus sunitinib for advanced renal-cell carcinoma. $N$ Engl $J$ Med. 2019;380:1103-1115. doi:10.1056/NEJMoa1816047

17. Escudier B, Eisen T, Stadler WM, et al. Sorafenib for treatment of renal cell carcinoma: final efficacy and safety results of the phase III treatment approaches in renal cancer global evaluation trial. $J$ Clin Oncol. 2009;27:3312-3318. doi:10.1200/JCO.2008.19.5511

18. Rini BI, Escudier B, Tomczak P, et al. Comparative effectiveness of axitinib versus sorafenib in advanced renal cell carcinoma (AXIS): a randomised phase 3 trial. Lancet. 2011;378:1931-1939. doi:10.1016/S0140-6736(11)61613-9

19. Motzer RJ, Nosov D, Eisen T, et al. Tivozanib versus sorafenib as initial targeted therapy for patients with metastatic renal cell carcinoma: results from a phase III trial. $J$ Clin Oncol. 2013;31:3791-3799. doi:10.1200/JCO.2012.47.4940

20. Motzer RJ, Escudier B, McDermott DF, et al. Nivolumab versus everolimus in advanced renal-cell carcinoma. $N$ Engl $J$ Med. 2015;373:1803-1813. doi:10.1056/NEJMoa1510665 
21. Choueiri TK, Escudier B, Powles T, et al. Cabozantinib versus everolimus in advanced renal cell carcinoma (METEOR): final results from a randomised, open-label, phase 3 trial. Lancet Oncol. 2016;17:917-927. doi:10.1016/S1470-2045(16)30107-3

22. Motzer RJ, Hutson TE, Glen H, et al. Lenvatinib, everolimus, and the combination in patients with metastatic renal cell carcinoma: a randomised, phase 2, open-label, multicentre trial. Lancet Oncol. 2015;16:1473-1482. doi:10.1016/S1470-2045(15) 00290-9

23. Molina AM, Hutson TE, Nosov D, et al. Efficacy of tivozanib treatment after sorafenib in patients with advanced renal cell carcinoma: crossover of a phase 3 study. Eur J Cancer. 2018;94:87-94. doi:10.1016/j.ejca.2018.02.009

24. Procopio G, Prisciandaro M, Iacovelli R, et al. Safety and efficacy of cabozantinib in metastatic renal-cell carcinoma: real-world data from an Italian managed access program. Clin Genitourin Cancer. 2018;16:e945-e51. doi:10.1016/j.clgc.2018.03.014

25. Yip SM, Wells C, Moreira R, et al. Checkpoint inhibitors in patients with metastatic renal cell carcinoma: results from the international metastatic renal cell carcinoma database consortium. Cancer. 2018;124:3677-3683. doi:10.1002/cncr.31595

26. Summary of opinion: Fotivda tivozanib hydrochloride monohydrate. Committee for Medicinal Products for Human Use. European MedicinesAgency;2017 Available from: https://www.ema.europa.eu/ en/documents/assessment-report/fotivda-epar-public-assessmentreport_en.pdf. Accessed April 18, 2019.
27. Nakamura K, Taguchi E, Miura T, et al. KRN951, a highly potent inhibitor of vascular endothelial growth factor receptor tyrosine kinases, has antitumor activities and affects functional vascular properties. Cancer Res. 2006;66:9134-9142. doi:10.1158/0008-5472.CAN-05-4290

28. Ferrara N, Gerber HP, LeCouter J. The biology of VEGF and its receptors. Nat Med. 2003;9:669-676. doi:10.1038/nm0603-669

29. Niwakawa M, Yamaguchi R, Onozawa Y, et al. Phase I study of highly selective inhibitor of VEGFR tyrosine kinase, tivozanib, in Japanese patients with solid tumors. Cancer Sci. 2013;104:1039-1044. doi:10.1111/cas.12197

30. Eskens FA, de Jonge MJ, Bhargava P, et al. Biologic and clinical activity of tivozanib (AV-951, KRN-951), a selective inhibitor of VEGF receptor-1, -2, and -3 tyrosine kinases, in a 4-week-on, 2-weekoff schedule in patients with advanced solid tumors. Clin Cancer Res. 2011;17:7156-7163. doi:10.1158/1078-0432.CCR-11-0411

31. Nosov DA, Esteves B, Lipatov ON, et al. Antitumor activity and safety of tivozanib (AV-951) in a phase II randomized discontinuation trial in patients with renal cell carcinoma. $J$ Clin Oncol. 2012;30:1678-1685. doi:10.1200/JCO.2011.35.3524

32. Hutson TE, Lesovoy V, Al-Shukri S, et al. Axitinib versus sorafenib as first line therapy in patients with metastatic renal-cell carcinoma: a randomised open-label phase 3 trial. Lancet Oncol. 2013;14:1287-1294. doi:10.1016/S1470-2045(13)70465-0

33. Fishman MN, Srinivas S, Hauke RJ, et al. Phase Ib study of tivozanib (AV-951) in combination with temsirolimus in patients with renal cell carcinoma. Eur J Cancer. 2013;49:2841-2850. doi:10.1016/j. ejca.2013.04.019

\section{Publish your work in this journal}

The International Journal of Nephrology and Renovascular Disease is an international, peer-reviewed open-access journal focusing on the pathophysiology of the kidney and vascular supply. Epidemiology, screening, diagnosis, and treatment interventions are covered as well as basic science, biochemical and immunological studies. The manuscript management system is completely online and includes a very quick and fair peer-review system, which is all easy to use. Visit http://www.dovepress.com/testimonials.php to read real quotes from published authors. 